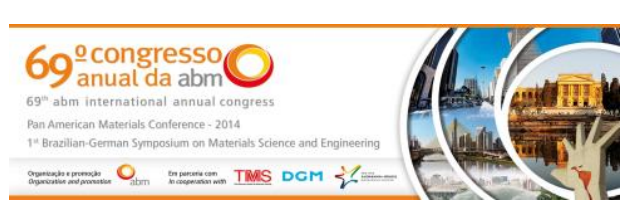

Tema: Diagramas de Fases e Transformações de Fases

\title{
PERFIL DE MICROSSEGREGAÇÃO DE UMA LIGA Ag-Cu SUBMETIDA À SOLIDIFICAÇÃO RÁPIDA*
}

Pollyana de Aragão Trigueiro ${ }^{1}$

Romulo Adolfo Heringer Ferreira ${ }^{2}$

\section{Resumo}

A distribuição de soluto durante a solidificação rápida de uma liga $\mathrm{Ag}-15 \%$ massaCu, foi obtida numericamente. No presente trabalho, utilizamos um modelo de difusão de entalpia e concentração médias, proposto anteriormente, para a solidificação de uma gota de liga binária (atomização). Nossos resultados mostram que a refusão da estrutura pode gerar um perfil de microssegregação em ligas solidificadas rapidamente bastante diferente dos que resultam da solidificação perto do equilíbrio. Palavras-chave: Solidificação rápida; Microssegregação; $\mathrm{Ag}-\mathrm{Cu}$; Simulação numérica.

\section{MICROSEGREGATION PROFILE OF A RAPID SOLIDIFIED Ag-Cu ALLOY}

\section{Abstract}

The microsegregation profile over the cross section of dendrite arms in a rapidly solidified Ag-15\%massCu alloy has been calculated numerically. In the present work we have used an average diffusive model proposed previously for solidification a binary alloy droplet (atomisation). Our results show that remelting can generate a microssegregation profile in rapidly solidified alloys quite different from those resulting of solidification near equilibrium.

Keywords: Rapid solidification; Microsegregation, $\mathrm{Ag}-\mathrm{Cu}$; Numerical modeling.

1 Aluna de Doutorado do Programa de Pós-Graduação em Ciência e Engenharia de Materiais, UFPB, João Pessoa, Paraíba, Brasil.

2 Professor Adjunto no Dep. de Engenharia de Materiais, UFPB, João Pessoa, Paraíba, Brasil.

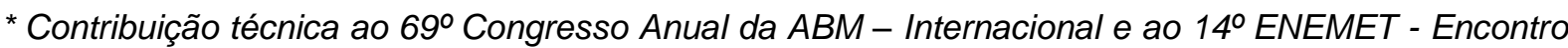
Nacional de Estudantes de Engenharia Metalúrgica, de Materiais e de Minas, 21 a 25 de julho de 2014, São Paulo, SP, Brasil.
} 


\section{INTRODUÇÃO}

Processos de solidificação rápida levam ao afastamento das condições de equilíbrio durante a solidificação de ligas, o que pode conduzir à formação de fases metaestáveis, à formação de materiais amorfos, supersaturação do sólido e refinamento espontâneo de grãos (causado pela refusão) [1].

Boettinger et al. [2] utilizaram uma técnica de solidificação rápida, através do tratamento a laser da superfície de uma chapa fina de um sistema $\mathrm{Ag}-15 \% \mathrm{Cu}$, para determinar o efeito que as condições de não-equilíbrio na interface de crescimento tem sobre a formação do perfil de microssegregação da liga binária $\mathrm{Ag}-\mathrm{Cu}$. Os autores mediram a concentração de soluto em pontos ao longo da seção transversal de braços de dendritas de peças resolidificadas como mencionado, de maneira que puderam traçar perfis de concentração ao longo de uma linha diametral. Os autores observaram perfis de concentração de soluto com um comportamento diferente do esperado. Algumas amostras, como a amostra do resultado mostrado na Figura (1), apresentaram uma região rica em soluto no centro dos braços de dendrita, diminuindo com o aumento do raio e voltando a crescer até a periferia. No presente trabalho a este comportamento será referido como "perfil em forma de W".

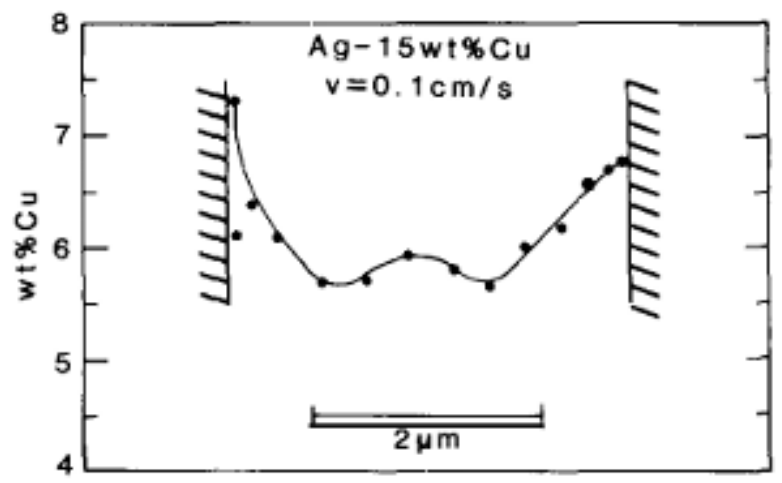

Figura 1. Medidas de concentração de soluto na seção transversal de um braço de dendrita feitas por Boettinger et al. [2].

O objetivo do presente trabalho foi de modelar situação equivalente àquela produzida por Boettinger et al [2] para uma liga $\mathrm{Ag}-15 \%$ massaCu, de maneira a tentar explicar as razões do perfil em forma de $\mathrm{W}$ medidos por eles. Para tanto, foi utilizado um modelo proposto Heringer $[3,4]$ escrito para solidificação de uma gota metálica esférica atomizada.

Os resultados obtidos sugerem que a causa do perfil em W é a refusão parcial do sólido supersaturado formado em um meio superresfriado.

\section{MATERIAIS E MÉTODOS}

\subsection{Abordagem numérica}

No experimento de Boettinger et al [2] foi utilizada uma técnica de solidificação rápida através do tratamento a laser da superfície de uma chapa fina do sistema Ag$15 \% \mathrm{Cu}$. Seus resultados mostram perfis de concentração para várias velocidades de solidificação como parâmetro.

No presente trabalho optou-se por um modelo físico diferente, porém similar ao experimento, mantendo a velocidade de solidificação como parâmetro de

\footnotetext{
* Contribuição técnica ao 69ำ Congresso Anual da ABM - Internacional e ao 14ํㅡㄹ ENEMET - Encontro Nacional de Estudantes de Engenharia Metalúrgica, de Materiais e de Minas, 21 a 25 de julho de 2014, São Paulo, SP, Brasil.
} 


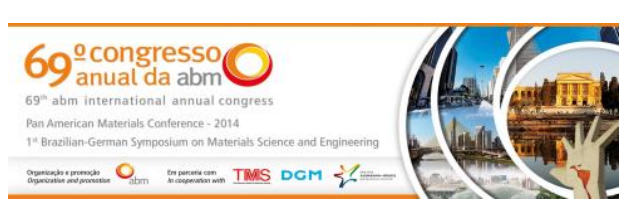

comparação. O modelo consiste na solidificação de um volume esférico (gota) metálico resfriado por convecção em um meio gasoso. As considerações iniciais e de contorno do modelo físico são as seguintes:

1. Inicialmente a temperatura e a concentração são conhecidas e uniformes na gota líquida;

2. Os campos de temperatura e concentração médios de soluto são de simetria esférica;

3. Só ocorre interação entre a gota e o meio gasoso;

4. No interior das gotas metálicas ocorre condução de calor e difusão de espécies químicas. As regiões formadas por sólido e líquido são consideradas uma mistura pastosa. As densidades do sólido e do líquido são consideradas iguais, para evitar fenômenos como contração na solidificação;

5. Na superfície da gota é aplicado um modelo de resfriamento de Newton com a definição de um coeficiente de transferência de calor por convecção, $h_{c}$. Para a transferência de massa, a superfície da gota é impermeável;

6. Consideramos nucleação única no centro da gota com crescimento esférico na zona pastosa, com crescimento a partir deste ponto. A zona pastosa é a região que contém o ponto de nucleação e é delimitada por uma superfície imaginária que toca as pontas das dendritas; a superfície, às vezes, é chamada de frente de solidificação e se move com a velocidade das pontas das dendritas.

As equações de conservação da energia e de soluto escritas para uma mistura das fases líquida e sólida constituem a base do modelo macroscópico. Para tanto, o meio foi caracterizado por médias, em um volume elementar representativo ( $\mathrm{VER}$ ), das propriedades termodinâmicas e das propriedades termofísicas, assim como das próprias equações de conservação. Foram definidas três regiões para a tomada das médias: a fase sólida, o líquido em contato com o sólido e o líquido livre, externo ao envelope do grão. A separação entre os dois líquidos é feita pela definição de um envelope de grão que contém tanto a fase líquida quanto a fase sólida e a morfologia do envelope é mais acessível do que as dendritas que ele contém. Uma aproximação pode ser feita considerando o envelope como sendo uma esfera.

As equações e propriedades médias que descrevem o meio em escala macroscópica são acopladas aos fenômenos caracterizados por ordens de grandeza microscópica através da temperatura e das frações mássicas da interface sólido/líquido no lado líquido $\left(w^{1 / s}\right)$ e no lado sólido $\left(w^{s / l}\right)$. Estes últimos são relacionados pelo diagrama de equilíbrio de fases à temperatura local. Nesta escala, foi utilizado o modelo de microssegregação baseado na equação de GulliverScheil [5]. Isto implica que no presente trabalho a difusão do soluto no sólido é negligenciada enquanto que no líquido presente entre as dendritas, é considerada difusão completa do soluto, de acordo com o modelo de Gulliver-Scheil [5].

A descrição matemática completa e o algoritmo numérico usados no presente trabalho podem ser encontrados em Heringer [3,4].

\subsection{Condições de Cálculo}

$\mathrm{Na}$ tabela (1) são definidas as propriedades termofísicas do sistema estudado e as condições gerais para os cálculos do modelo. Os resultados são considerados a partir de superresfriamentos iniciais, $\Delta \mathrm{T}=5 \mathrm{~K}, \Delta \mathrm{T}=10 \mathrm{~K}$ e $\Delta \mathrm{T}=30 \mathrm{~K}$. Alguns parâmetros são mantidos inalterados, como o tamanho da gota metálica, $R=125$ $\mu \mathrm{m}$, o coeficiente de transferência de calor do fluido que envolve a gota, $\mathrm{h}_{\mathrm{c}}=490 \mathrm{~W} /$

* Contribuição técnica ao 69 Congresso Anual da ABM - Internacional e ao 14ํㅡㄹ ENEMET - Encontro Nacional de Estudantes de Engenharia Metalúrgica, de Materiais e de Minas, 21 a 25 de julho de 2014, São Paulo, SP, Brasil. 


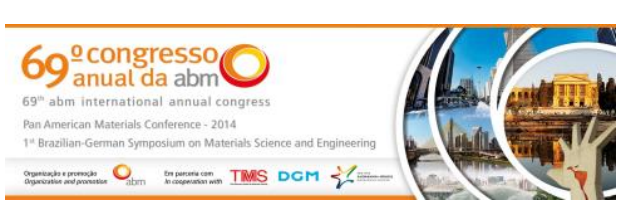

$\mathrm{m}^{2} . \mathrm{K}$ e a composição inicial $\mathrm{w}_{\mathrm{I}}=15 \%$ massa $\mathrm{Cu}$. Para o modelo numérico usado foi adotada uma malha de 40 volumes, para uma gota de $250 \mu \mathrm{m}$ de diâmetro. A resolução do modelo se baseia no método dos volumes finitos e a tomada das médias das grandezas descritivas é feita no volume elementar representativo para uma mistura sólido/líquido.

Tabela 1. Propriedades termofísicas do sistema $\mathrm{Ag}-\mathrm{Cu}$

\begin{tabular}{|l|l|l|l|l|}
\hline $\begin{array}{l}\text { Propriedad } \\
\mathrm{e}\end{array}$ & Valor & Unidade & Descrição & Referência \\
\hline $\mathrm{D}_{\mathrm{l}}$ & $2,1 \times 10^{-9}$ & $\mathrm{~m}^{2} \cdot \mathrm{s}^{-1}$ & $\begin{array}{l}\text { Coeficiente de difusão no } \\
\text { líquido }\end{array}$ & {$[2]$} \\
\hline $\mathrm{Tm} \Gamma$ & $1,5 \times 10^{-7}$ & $\mathrm{~K} \cdot \mathrm{m}$ & Capilaridade & {$[2]$} \\
\hline $\mathrm{L}$ & $\begin{array}{l}167472000 \\
0\end{array}$ & $\mathrm{~J} . \mathrm{m}^{-3}$ & Entalpia de fusão & {$[6]$} \\
\hline $\mathrm{K}$ & 293,076 & $\mathrm{~W} \cdot \mathrm{m}^{-1} \cdot \mathrm{K}^{-1}$ & Condutividade térmica & {$[6]$} \\
\hline $\mathrm{C}$ & 405,697 & $\mathrm{~J}_{\mathrm{Kg}} \mathrm{Kg}^{-1} \cdot \mathrm{K}^{-1}$ & Calor específico & {$[6]$} \\
\hline $\mathrm{D}_{0}$ & $1,22 \times 10^{-7}$ & $\mathrm{~m}^{2} \cdot \mathrm{s}^{-1}$ & Termo pré-exponencial & {$[7]$} \\
\hline $\mathrm{Q}$ & 41800 & $\mathrm{~J} \mathrm{~mol}^{-1}$ & Coeficiente de ativação & {$[7]$} \\
\hline $\mathrm{T}_{\mathrm{f}}$ & 961,78 & ${ }^{\circ} \mathrm{C}$ & $\begin{array}{l}\text { Temperatura de fusão da } \mathrm{Ag} \\
\text { pura }\end{array}$ & {$[8]$} \\
\hline $\mathrm{T}_{\mathrm{E}}$ & 779,1 & ${ }^{\circ} \mathrm{C}$ & Temperatura eutética & {$[8]$} \\
\hline $\mathrm{T}_{\infty}$ & 100 & ${ }^{\circ} \mathrm{C}$ & $\begin{array}{l}\text { Temperatura do gás } \\
\text { envolvente }\end{array}$ & {$[3]$} \\
\hline $\mathrm{A}$ & $3,572 \times 10^{-6}$ & $\mathrm{~m} \cdot \mathrm{s}^{-1}$ & $\begin{array}{l}\text { Constante de } \\
\text { proporcionalidade }\end{array}$ & {$[3]$} \\
\hline $\mathrm{n}$ & 2,7 & {$[1]$} & Expoente & {$[3]$} \\
\hline
\end{tabular}

\section{RESULTADOS E DISCUSSÃO}

\subsection{Caminhos de solidificação}

As condições para os cálculos são as apresentadas na Tabela (1), as medidas são feitas em três posições ( $R=0, R=1 / 2$ e $R=1)$, para solidificação de uma gota metálica num sistema $\mathrm{Ag}-15 \% \mathrm{Cu}$, com um superresfriamento inicial, $\Delta \mathrm{T}=30 \mathrm{~K}$.

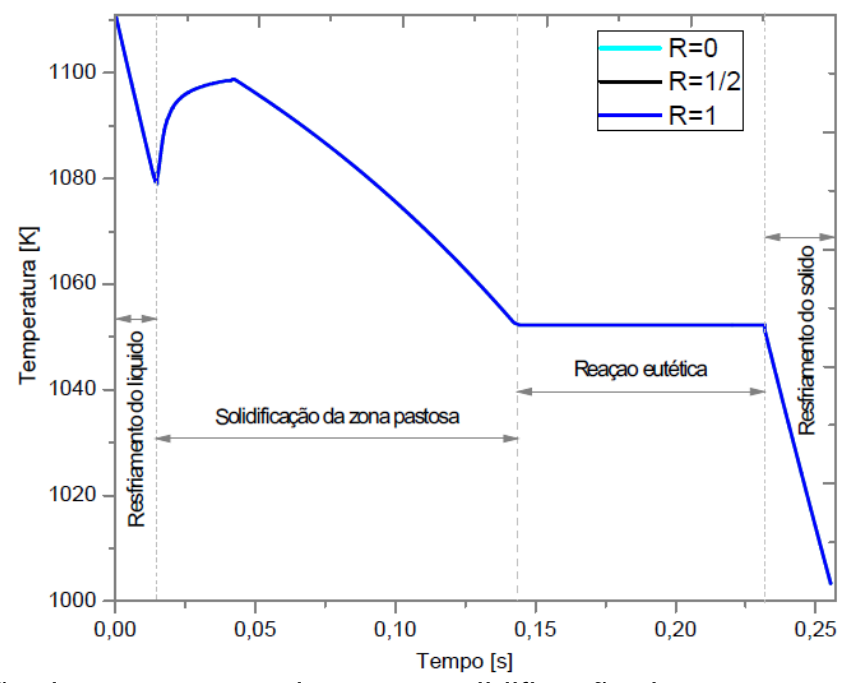

Figura 2. Evolução da temperatura durante a solidificação da gota com diâmetro de $250 \mu \mathrm{m}$.

\footnotetext{
* Contribuição técnica ao $69^{\circ}$ Congresso Anual da ABM - Internacional e ao 14ํㅡㄹ ENEMET - Encontro Nacional de Estudantes de Engenharia Metalúrgica, de Materiais e de Minas, 21 a 25 de julho de 2014, São Paulo, SP, Brasil.
} 


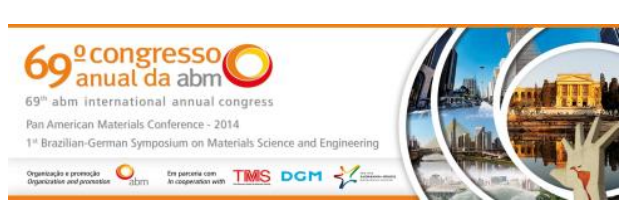

Assim que a nucleação ocorre no centro da gota, ou seja, em $\mathrm{R}=0$, um aumento de temperatura da zona pastosa é previsto, de acordo com a Figura (2). O sistema é rapidamente aquecido como um todo. Tal reaquecimento, referido como recalescência, é, devido ao calor latente libertado pela crescente zona pastosa, que não é removido por todo o fluxo de calor aplicado na superfície da gota.
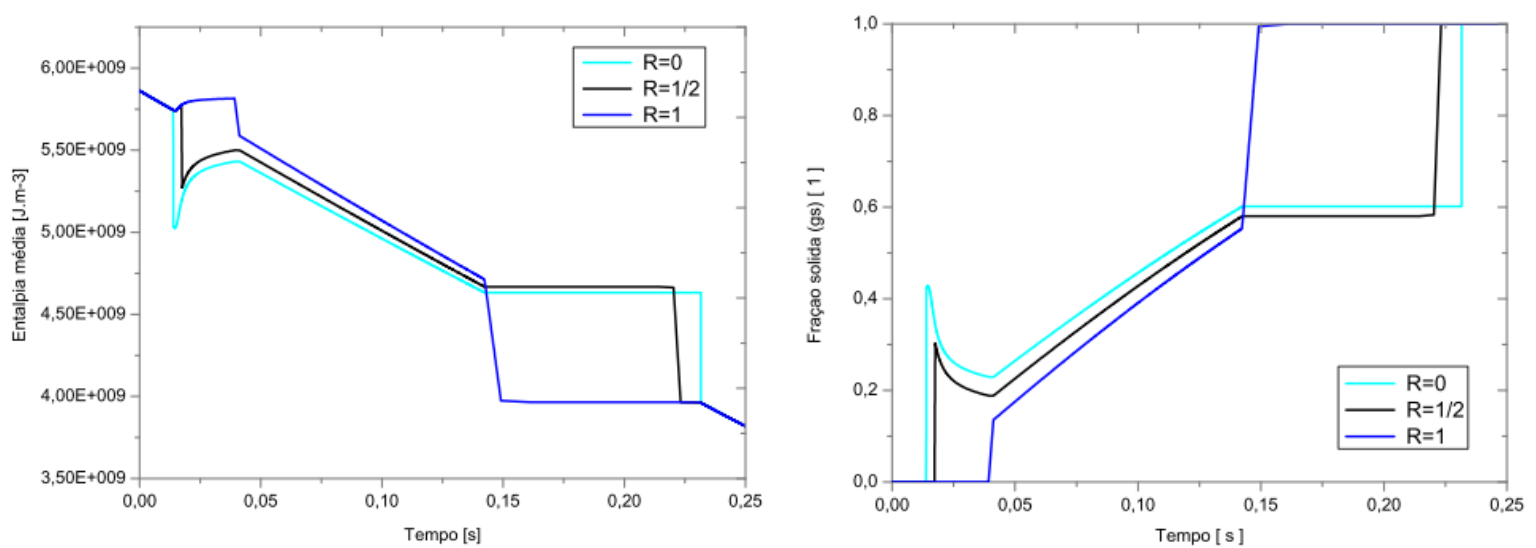

Figura 3. Evolução da entalpia (a) e da fração sóllida (b) durante a solidificação de um sistema $\mathrm{Ag}-\mathrm{Cu}$ nas posições indicadas.

$\mathrm{Na}$ Figura (2) observa-se uma queda na temperatura nos primeiros milésimos de segundos, quando a liberação de entalpia local ainda é menor que a extração de calor para fora do sistema, pois o sólido formado ainda é pequeno, logo em seguida a temperatura volta a aumentar, quando a energia no interior do sistema é maior que o fluxo de calor externo, a solidificação já começou e durante este processo a entalpia diminui e a fração sólida aumenta, de acordo com a Figura (3), em contrapartida da diminuição da temperatura neste mesmo período, até que a gota esteja totalmente solidificada. Essa diminuição da temperatura conduz a um aumento no superresfriamento e consequentemente um aumento na velocidade da frente de solidificação. Com o avanço da frente de solidificação e aumento do volume da zona pastosa, temos uma diminuição da entalpia, chegando a um mínimo, seguida de um aumento abrupto de entalpia de fusão liberada, que é maior que o fluxo de calor convectivo no fluido que envolve a gota e neste mesmo período podemos observar um aumento seguido de uma diminuição da fração de sólido volumétrica, durante a recalescência.

\subsection{Perfil de microssegregação}

As frações mássicas da interface sólido/líquido no lado líquido $\left(w^{1 / s}\right)$ e no lado sólido $\left(w^{s / l}\right)$ são calculadas a partir do diagrama de fases à temperatura local. Estes valores não são valores médios, sendo assim, $\mathrm{w}^{\mathrm{s} / \mathrm{l}}$ em cada ponto representa o valor da concentração naquele ponto sólido. D A partir dos resultados numéricos são conhecidos os históricos de $\mathrm{w}^{\mathrm{s} / \mathrm{l}} \mathrm{em}$ cada ponto nodal durante todo o processo de solidificação.

No entanto, uma vez que a complexa morfologia das dendritas não é conhecida ou levada em conta no presente modelo, não são conhecidas as dimensões de nenhum braço de dendrita em particular. Sendo assim, a princípio não seria possível atribuir um valor de concentração a uma determinada posição em um braço de dendrita, como é o caso nos resultados experimentais de Boettinger et al [2]. Esta dificuldade

\footnotetext{
* Contribuição técnica ao $69^{\circ}$ Congresso Anual da ABM - Internacional e ao 14ํㅡㄹ ENEMET - Encontro Nacional de Estudantes de Engenharia Metalúrgica, de Materiais e de Minas, 21 a 25 de julho de 2014, São Paulo, SP, Brasil.
} 


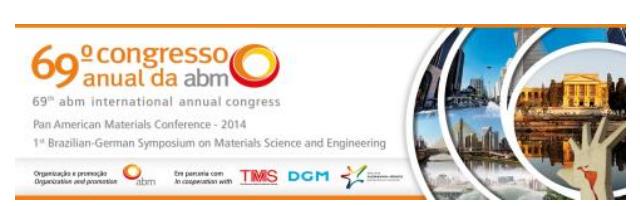

é contornada usando a fração de sólido $\mathrm{g}^{\mathrm{s}}$ como a posição relativa da periferia do braço de dendrita plotando uma curva $\left(g^{s}, w^{s / l}\right)$. Sabe-se que em ligas hipoeutéticas o crescimento dos braços de dendrita seguido de sua maturação ocorre até o limite da fração da primeira fase formada (em geral, fase $\alpha$ ). Sendo assim, o valor de $\mathrm{g}^{\mathrm{s}}$ correspondente ao início da transformação eutética em uma dada posição que pode ser tomado como o limite máximo da dimensão relativa do braço de dendrita naquele ponto. A Figura 4(a) mostra curvas de $w^{s / l}$ em função de $g^{s}$ para uma dada posição para três superresfriamentos iniciais. Pode-se notar que na figura 4(a) levou-se em conta $g^{s}$ limitados pelo início da fase eutética.

A fim de representar convenientemente a dimensão relativa da seção transversal do braço de dendrita, foi definida uma fração de fase $\alpha, g^{\alpha}$, fazendo o quociente entre a fração de sólido $g^{s}$ e o valor local da da fração de sólido no início da fase eutética (ou seja a fração final de fase a naquela posição). Com isso pode-se construir as curvas da figura 4(b), com uma quantidade adimensional variando de 0 a 1.
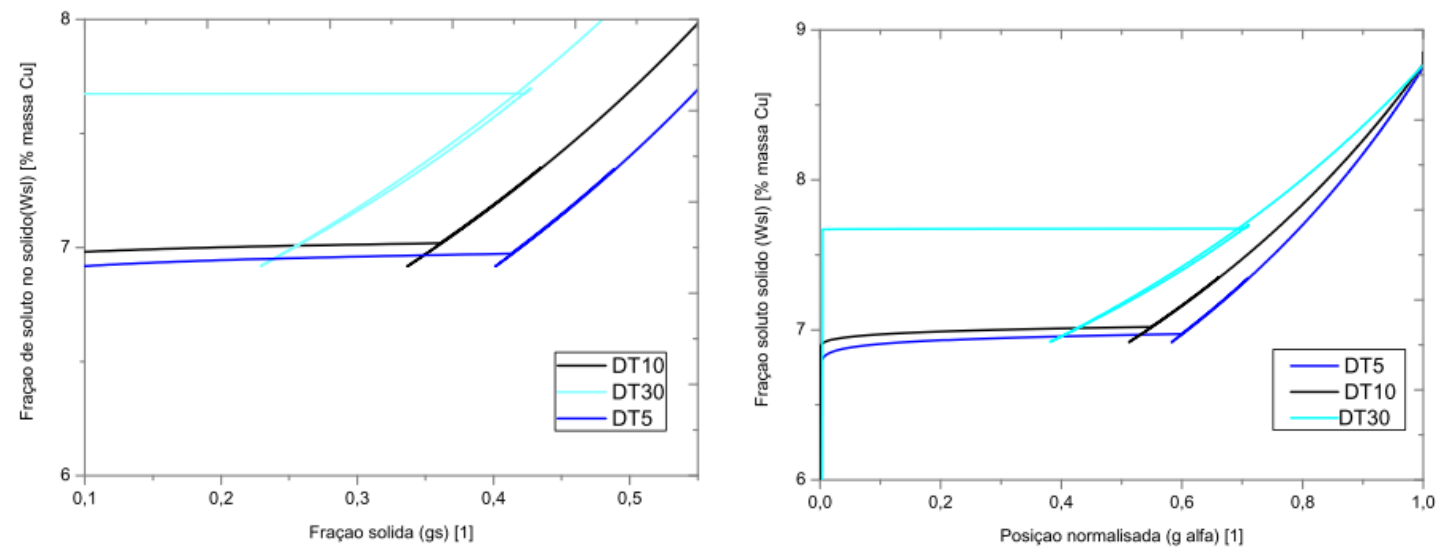

Figura 4. Evolução da fração mássica de soluto em relação à fração sólida (a) e em uma posição normalizada, sólido $g_{\alpha}$, (b) para os três superresfriamentos. O recuo observado é devido à refusão.

O histórico de $W^{\text {s/l }}$ mostrado na Figura (4) não representa o perfil de microssegregação observável na peça solidificada. Os perfis de microssegregação são mostrados na Figura (5). Trata-se dos mesmos dados das curvas da Figura (4), porém, de onde se desconsiderou os pontos correspondentes ao sólido consumido pela refusão e que, portanto, não estaria mais presente na peça solidificada. Com isso, na Figura (5) tem-se a representação do perfil de microssegregação na microestrutura.

Pode-se observar, na Figura (5), que a região central é equivalente a um patamar com queda abrupta na fronteira que marca o limite até onde o primeiro sólido formado em cada ponto foi refundido. Esta variação seria menos súbita caso a difusão de soluto no sólido fosse levada em conta. A partir de um líquido superresfriado, o soluto é rejeitado e se acumula na frente de solidificação e esse acúmulo de soluto forma um líquido supersaturado e a distribuição de soluto no sólido é controlada pela cinética de solidificação [9], onde a partição de soluto depende da velocidade de crescimento da interface. Com o aumento da temperatura ocorre a recalescência e a refusão local do sólido a (dendritas). Após a recalescência a temperatura do sistema diminui e o avanço da frente de solidificação se dá em velocidades mais baixas e o soluto é difundido no líquido para distâncias maiores.

\footnotetext{
* Contribuição técnica ao $69^{\circ}$ Congresso Anual da ABM - Internacional e ao 14ํㅡㄹ ENEMET - Encontro Nacional de Estudantes de Engenharia Metalúrgica, de Materiais e de Minas, 21 a 25 de julho de 2014, São Paulo, SP, Brasil.
} 

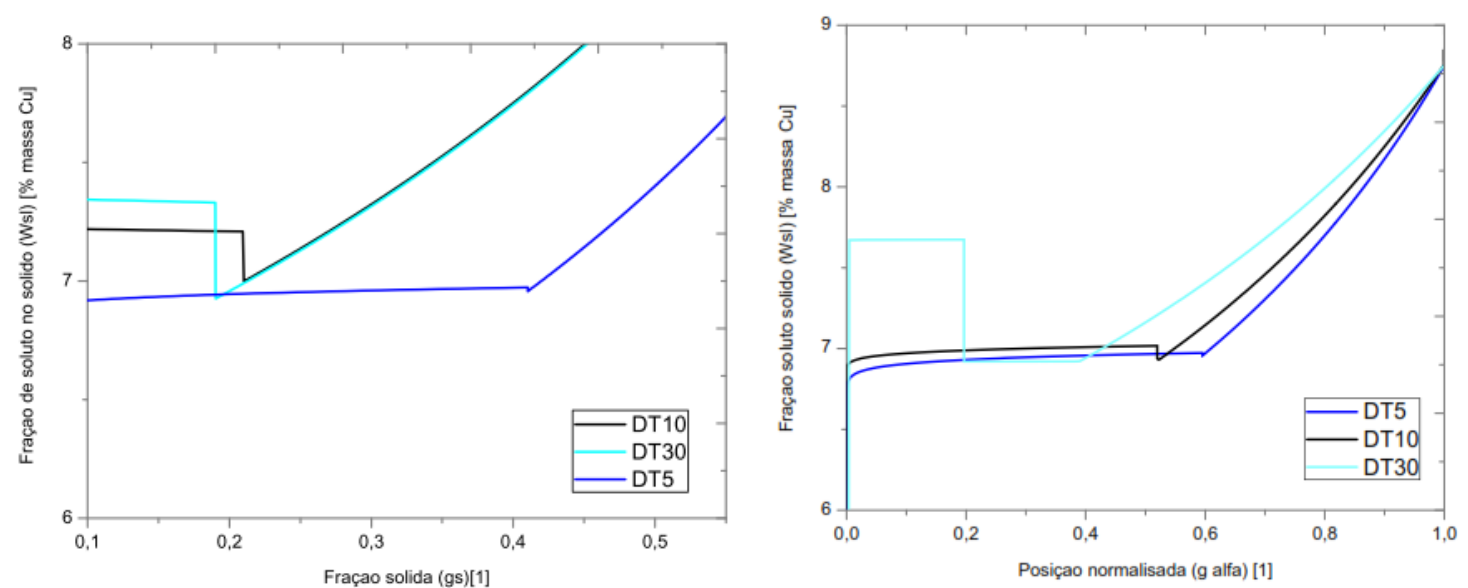

Figura 5. Perfil de distribuição de soluto (microssegregação) de um sistema $\mathrm{Ag}-15 \% \mathrm{Cu}$ solidificado rapidamente, em relação à fração sólida (a) e em uma posição normalizada, sólido ga, (b).

Os resultados obtidos sugerem que a causa do perfil em W é a refusão parcial do sólido supersaturado formado em um meio superresfriado. O sólido formado em um meio superresfriado consegue dissolver mais soluto do que em condições próximas do equilíbrio. Quanto maior o superresfriamento maior a velocidade de solidificação e, portanto, maior a taxa de liberação de entalpia de fusão. Se a transferência de calor não fizer face à taxa de liberação de entalpia de fusão, haverá aumento de entalpia e de temperatura do meio (recalescência). Nestas condições o sólido inicialmente formado torna-se supersaturado e é, portanto, refundido. A refusão continua até que a entalpia volte a diminuir (por transferência de calor). A partir dai a solidificação é retomada, porém com superresfriamento menor gerando sólidos com concentrações de soluto menores do que aqueles de antes da refusão. Esta transição, entre fim da refusão e retomada solidificação, corresponde à região de concentrações menores que à meio raio do braço de dendrita observado por Boettinger et al [2]. A solidificação continua com menor velocidade. A concentração do sólido volta a crescer à medida que a entalpia e temperatura diminuem, em acordo com o diagrama de equilíbrio de fases, como esperado nos processos de solidificação à baixa velocidade.

\section{CONCLUSÃo}

O modelo usado no presente trabalho foi aplicado para o cálculo da distribuição de soluto no sólido formado. Em comparação com a literatura, encontramos um perfil não-monotônico de microssegregação, onde existe uma região central rica em soluto, em velocidades próximas aos experimentos de Boettinger et al [2]. Os resultados obtidos sugerem que a causa do perfil em forma de $W$ é a refusão parcial do sólido supersaturado formado em um meio superresfriado.

\section{Agradecimentos}

À CAPES pelo financiamento parcial deste trabalho (bolsa de Mestrado); ao Programa de Pós-Graduação em Ciência e Engenharia de Materiais da UFPB; à equipe do Laboratório de Modelagem em Materiais - L2M/UFPB.

\footnotetext{
* Contribuição técnica ao $69^{\circ}$ Congresso Anual da ABM - Internacional e ao 14ํㅡㄹ ENEMET - Encontro Nacional de Estudantes de Engenharia Metalúrgica, de Materiais e de Minas, 21 a 25 de julho de 2014, São Paulo, SP, Brasil.
} 
